\title{
An analysis of the 2016 Hitomi breakup event
}

\author{
Sven Flegel ${ }^{1 *} \mathbb{D}$, James Bennett ${ }^{1}$, Michael Lachut ${ }^{1}$, Marek Möckel ${ }^{1}$ and Craig Smith ${ }^{2}$
}

\begin{abstract}
The breakup of Hitomi (ASTRO-H) on 26 March 2016 is analysed. Debris from the fragmentation is used to estimate the time of the event by propagating backwards and estimating the close approach with the parent object. Based on this method, the breakup event is predicted to have occurred at approximately 01:42 UTC on 26 March 2016. The Gaussian variation of parameters equations based on the instantaneous orbits at the predicted time of the event are solved to gain additional insight into the on-orbit position of Hitomi at the time of the event and to test an alternate approach of determining the event epoch and location. A conjunction analysis is carried out between Hitomi and all catalogued objects which were in orbit around the estimated time of the anomaly. Several debris objects have close approaches with Hitomi; however, there is no evidence to support the breakup was caused by a catalogued object. Debris from both of the largest fragmentation events - the Iridium 33-Cosmos 2251 conjunction in 2009 and the intentional destruction of Fengyun 1C in 2007-is involved in close approaches with Hitomi indicating the persistent threat these events have caused in subsequent space missions. To quantify the magnitude of a potential conjunction, the fragmentation resulting from a collision with the debris is modelled using the EVOLVE-4 breakup model. The debris characteristics are estimated from two-line element data. This analysis is indicative of the threat to space assets that mission planners face due to the growing debris population. The impact of the actual event to the environment is investigated based on the debris associated with Hitomi which is currently contained in the United States Strategic Command's catalogue. A look at the active missions in the orbital vicinity of Hitomi reveals that the Hubble Space Telescope is among the spacecraft which may be immediately affected by the new debris.
\end{abstract}

Keywords: Fragmentation, Debris, Spacecraft, Kessler syndrome

\section{Background}

To date, over 250 objects have broken up in Earth orbit (Johnson et al. 2008; Flegel et al. 2011). Breakups are especially critical whenever they occur in highly utilised orbits such as the $800-\mathrm{km}$-altitude band or the geostationary orbit (GEO) region. A collision with an active spacecraft may not only lead to the premature termination of the satellite's mission, but also create additional debris which in turn may collide with other objects. The process wherein debris from collisions becomes the main driver behind the creation of new debris is known as the 'Kessler syndrome' and may already be a reality (Kessler

\footnotetext{
*Correspondence: svenflegel@serc.org.au

1 Space Environment Research Centre (SERC) Limited, Weston Creek, ACT 2611, Australia

Full list of author information is available at the end of the article
}

1991; Inter-Agency Space Debris Coordination Committee-Working Group 2 2013).

A well-founded analysis of the immediate and longterm impacts of a breakup on the environment requires knowledge not only of the amount of debris which has been created in the event, what orbits these are on and how long they may remain there, but also of the current state of the environment. The largest, publicly accessible catalogue of objects on Earth orbits is being maintained by the United States Strategic Command (USSTRATCOM). Of the roughly 17,800 in-orbit objects currently contained in this catalogue, 11 debris objects have been associated with Hitomi's breakup. As the spacecraft's orbit inclination was only about $30^{\circ}$ and the orbital altitude was below $600 \mathrm{~km}$, many high-accuracy groundbased sensors cannot observe or track these objects simply because they never appear above their horizon. 
Although it is not expected that significant debris from the event should be missing from the catalogue, an analysis of the recorded debris orbits is nevertheless performed to assess whether the current understanding of the cause of the event is in line with the number of objects that are being tracked.

The current paper starts out with an outline of the events leading up to the fragmentation of Hitomi as published in media reports by Japan's Aerospace Exploration Agency (JAXA). The time at which the satellite broke up, the on-orbit location and the magnitude of the event are analysed. The debris environment in the vicinity of Hitomi is then assessed by calculating close conjunctions between Hitomi and debris with known orbits. Modelling the fragmentation of Hitomi resulting from a collision with another object deepens the understanding of the risks associated with such events and introduces an alternate possible cause for the breakup. The section also introduces another possible cause for the breakup of Hitomi which cannot be dismissed based on the current evidence. In the final section, the impact of the debris created in the actual breakup of Hitomi on the debris environment and on active spacecraft in its vicinity is investigated.

\section{Event description}

On 31 May 2016, JAXA published a detailed report describing events leading up to the breakup of Hitomi (JAXA 2016). JAXA estimated the breakup to have occurred around 1:37 UTC on March 26 by backpropagating the debris positions. This assessment is just 5 min earlier than the epoch published by the United States Joint Space Operations Centre (JSpOC): $1: 42 \mathrm{UTC} \pm 11 \mathrm{~min}$. The report named four major 'mechanisms' which are suspected to have lead to the spacecraft being spun up by its attitude control system (ACS) whereby both solar panels and the extensible optic bench (EOB) are likely to have broken off: (1) the ACS indicated the attitude state of the spacecraft as rotating when it was not. The reaction wheel (RW) was then activated to counteract the rotation which caused the spacecraft to start spinning. (2) Unloading of the RW by the magnetic torquer did not work properly. (3) Once the spacecraft attitude situation had been determined as critical, ACS switched to the Safe Hold mode wherein attitude thrusters were activated to return the spacecraft to a steady attitude. After the solar arrays and the EOB had been extended, new thruster control parameters had been uploaded to the spacecraft to account for the changes in centre of mass and moment of inertia. These parameters had, however, not been tested prior to uploading and turned out to be inaccurate. Simulations of the spacecraft attitude with the updated ACS control parameters which were performed after the event showed that the thrusting would have caused the spacecraft attitude to respond in an unexpected manner and likely increased the spacecraft spin rate further. (4) Angular rates derived from ground-based observations of Hitomi were then fed into finite element method simulations to test for structural response. Within the simulations, material limits were surpassed for both solar panels and for the EOB. The ACS-induced spinning up of the spacecraft has therefore been established as a likely cause for the breakup of Hitomi.

Within the current section, the event epoch and location are derived independently of the published results by JAXA and JSpOC. The event magnitude is then assessed based on the distribution of the orbits from the 11 catalogued debris. If the orbits suggest that a high-energy event occurred, there should be many more debris than the objects which have currently been identified from the event.

\section{Estimate of the time of the Hitomi breakup}

All of the debris created from the breakup event was used to estimate the time of the breakup by comparing their backwards propagated orbits with that of Hitomi. The time where the orbits are at their closest point can give an indication of the event time. Only valid two-line elements (TLEs) (i.e., removing the erroneous Hitomi ones) after the event were used to estimate the expected time of the breakup event. Figure 1 (left) shows a histogram for the time estimates for the orbit close approaches of Hitomi and Hitomi debris objects for the period around 26 March 2016 00:00:00 UTC. Overlaid on the plot is a normal probability density function fit to the data. The time interval 01:00-02:00 UTC on 26 March 2016 contains the most close approach estimates. The right-hand plot in Fig. 1 shows a histogram of the close approach times for 01:00-02:00 UTC on 26 March 2016. Fitting a normal distribution allows us to estimate the time of the breakup event. This gives a mean event time as 26 March 2016 01:42 UTC (26 March 2016 10:42 JST) and expected to fall within 01:42 $\pm 14 \mathrm{~min}$ ( $3 \sigma$ interval).

\section{Event location}

The event position is directly linked to the event epoch. Using SGP4 to estimate the on-orbit location of Hitomi at the time of the event yields an argument of latitude of $u=\omega+v=35^{\circ} \pm 52.53^{\circ}$ ( $\omega$ is the argument of perigee, and $v$ the true anomaly).

Orbit shape and orientation in inertial space change when a perturbing force acts on it. The manner in which they change depends on the force's direction and magnitude, the initial orbit, the argument of latitude and true anomaly at which it is applied. It should be possible to 

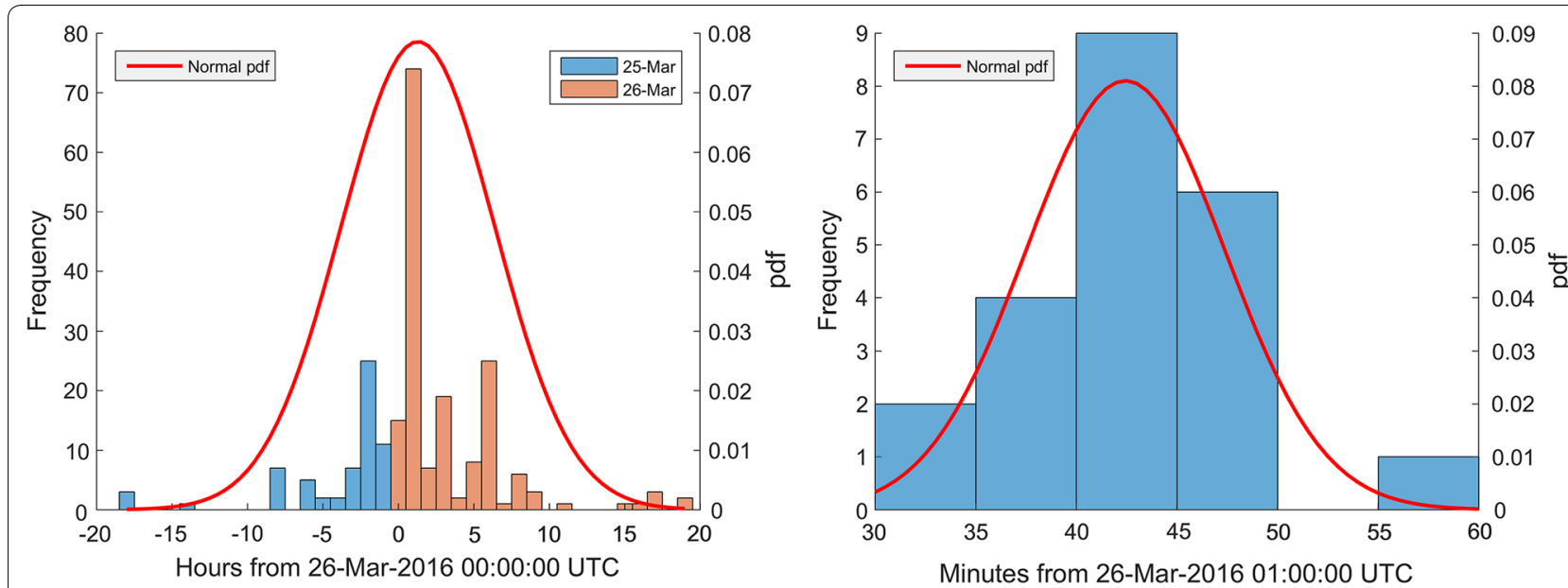

Fig. 1 Histogram and probability density function plot for the estimated breakup event times around 26 March 2016 00:00:00 UTC (left). Histogram and probability density function plot for the estimated breakup event times during 01:00-02:00 UTC on 26 March 2016 (right)

analyse the change in osculating orbital elements around the time of the event to determine the likely on-orbit position of Hitomi when the event occurred. Obtaining a solution in this manner may be helpful in confirming, refuting or even refining the solution from the previous close conjunction analysis.

The Gaussian form of the variation of parameters equations (Gaussian VoP) gives the time derivative of each orbit parameter based on three orthogonal accelerations in the RSW system [see for example Section 9.3.2 in Vallado and McClain (2013)]. For small accelerations, the equations can be integrated by keeping all orbit parameters fixed. The resulting relations give the total change in each parameter as the function of the change in velocity in each of the orthogonal RSW directions. Within these equations, the orbit shape and in-plane orientation are influenced by accelerations which are in the orbital plane; the orientation of the orbit plane in inertial space is only affected by accelerations normal to that plane. As the orbit changes encountered in the case of Hitomi are not small, the applicability of the method is first tested on simulated fragmentation events and then applied to the current event.

Applicability to fragmentation analyses Four main factors impact the accuracy of the method's outcome: (1) the method requires knowledge of the change in orbit parameters induced during the event. As the parameters evolve differently for each object, an estimate of the event epoch must be available beforehand. Unless this is included as a solve-for parameter, the accuracy with which the event epoch is known before can potentially impact the accuracy of this method's result. (2) The orbit data itself usually contains non-negligible inaccuracies. (3) The orbit parameters at the event epoch are obtained through propagation using a tool with a given accuracy. (4) Finally, the method relies on changes in the orbit parameters to be small.

In the following, only the basic applicability of the method to low-energy fragmentation events such as the one currently being analysed along with its potential accuracy and precision is assessed. This is done by applying the method to simulated debris clouds at the event epoch. In this manner, uncertainties are limited to inaccuracies at the machine level which are introduced mainly by the transformation of the state vectors into Keplerian elements.

Four test cases are created. The first test case $\mathbf{T} \mathbf{1}$ is based on preliminary results from the Gaussian VoP method applied to the breakup of Hitomi. The resulting velocity changes in RSW coordinates at the time of the event are translated into a covariance matrix for the velocity. The values in the matrix are increased by a factor 10 to allow for outliers to be adequately covered within the simulation. To attain statistical significance, 1000 debris particles are created at the true anomaly location $v=87.21^{\circ}$. These particles are all co-located and have instantaneous velocity vectors which cover the volume of values derived from Hitomi's debris. The state vectors of these particles are then transformed into Keplerian elements and processed by the Gaussian VoP method. The same methodology is applied to three more test cases. The second test $\mathbf{T} \mathbf{2}$ is identical to the first test case with the exception that the location of the event is moved to $v=20.00^{\circ}$. The two final test cases $\mathbf{T} 3$ and T4 use the same true anomaly locations as cases one and two but assume an initial orbit eccentricity of 0.05 instead of $\approx 0.0009$. It is found that solutions generally do not converge, if the change in argument of perigee due to 
the event exceeds roughly $20^{\circ}$. The absolute value of the residual error for all remaining particles is plotted against the true anomaly of the solution in Fig. 2. It can be seen that the true solution can be found by calculating the mean of values with residual errors below 0.0001. Assuming a higher threshold requires manual pre-screening of the results. For the most relevant test case $\mathbf{T 1}$, the resulting accuracy is worst which can be attributed to the parent orbit being near-circular and the event location near $v=90^{\circ}$. Using the solution of particles with absolute residual errors lower than 0.001 , the mean and standard deviation become: $v=88.18^{\circ} \pm 2.86^{\circ}$. The true value is therefore contained in the 1- $\sigma$ confidence interval, and the mean value is accurate to within less than $1^{\circ}$ of the true value.

Application to Hitomi The osculating orbit at the instances before and after the breakup is required for the current analysis. The former is obtained by propagating the last available TLE from before the event to the estimated event epoch 26 March 01:42 UTC using the Simplified General Perturbations 4 (SGP4) method. For each fragment, five successive TLEs are propagated back in time to the event epoch. The TLEs from the two objects 41438 and 41443 are omitted as they decayed within the first month after the event. For the further analysis, also the TLE from objects 41440, 41441 and 41446 are disregarded as their argument of true anomaly changed by more than $20^{\circ}$ through the event. This is in excess of what was established in the method testing to exceed the requirement of parameter changes to remain small. As Hitomi's orbit eccentricity was on the order of only $10^{-3}$, the argument of perigee changes very quickly. This causes the true anomaly in the days surrounding the event to remain within the interval $\left[-40^{\circ}, 100^{\circ}\right]$. Only those solutions from the Gaussian VoP analysis which are in this region and have converged to an absolute solution error of $1 \times 10^{-3}$ are considered. The solutions from TLEs of the main body, which has the 'North American Aerospace Defense Command' (NORAD) ID 41337, do not contribute to the overall result since their best absolute error is at $2 \times 10^{-3}$. The results based solely on its solutions are given independently nevertheless as its TLEs and the results obtained from them exhibit the greatest consistency.

The left plot within Fig. 3 shows the distribution of results from all valid TLEs without the main body. The evolution of true anomaly of the main body for the $\pm 6 \sigma$ interval surrounding March 26, 01:42 UTC is obtained by propagating the last valid TLE prior to the event forward in time. It is shown in the centre right plot in Fig. 3. The normal probability density function (PDF) above the true anomaly evolution represents the solution for the predicted event epoch from the previous section. The normal PDF derived from the simultaneous solving of the Gaussian VoP equations for the true anomaly is given to the right. The mean and standard deviation of the normal distribution based on the TLE's of all debris are $v=83.69^{\circ} \pm 6.23^{\circ}$. The solution using only the TLE from Hitomi's main body is $v=82.72^{\circ} \pm 0.08^{\circ}$. Both solutions are within a degree of one another. This is on the order of the accuracy which can be expected of the method when applied to the specific case of Hitomi (see Test Case T1).

Projecting the solutions from the Gaussian VoP method onto the time-dependent true anomaly of the main body shows two possible locations in the $\pm 6 \sigma$ interval surrounding the estimated event epoch: one is located at around $+11 \mathrm{~min}$ and the other at $+20 \mathrm{~min}$ after the estimated event epoch. Here, only the solution closer to the estimated event time is considered. The PDFs from the debris close approach and from the Gaussian VoP analyses can be combined to form a new estimate of the event epoch. It is obtained by multiplying the normalised probability for the individual solutions at each true
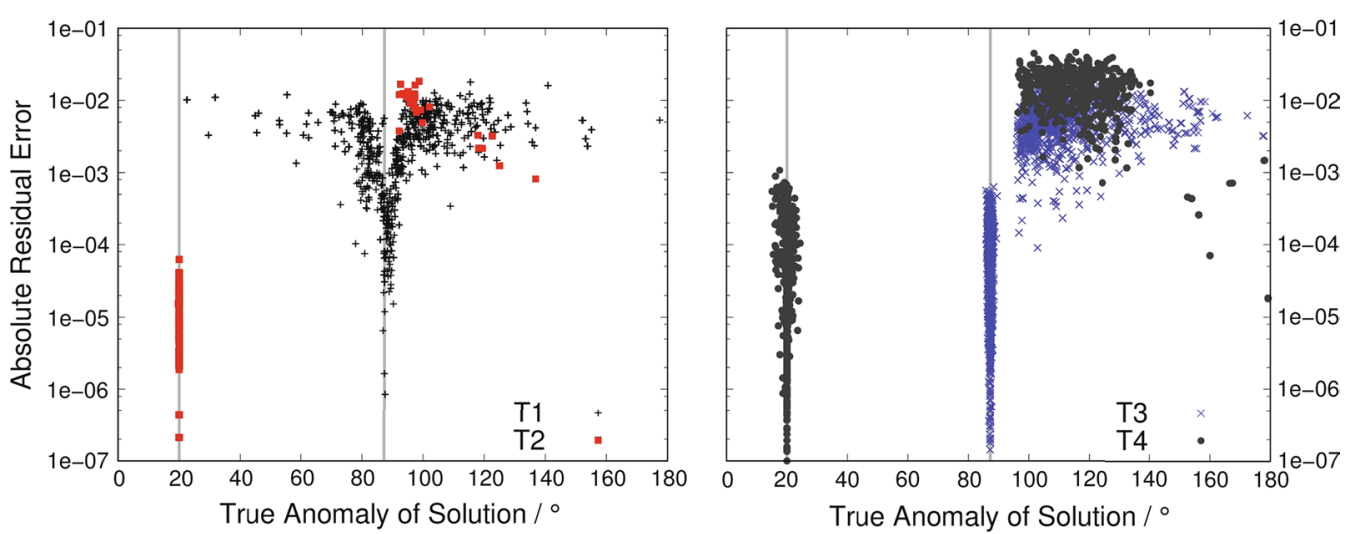

Fig. 2 Solution of the Gaussian VoP method to four simulated satellite fragmentations. The grey lines indicate the true location of each event 

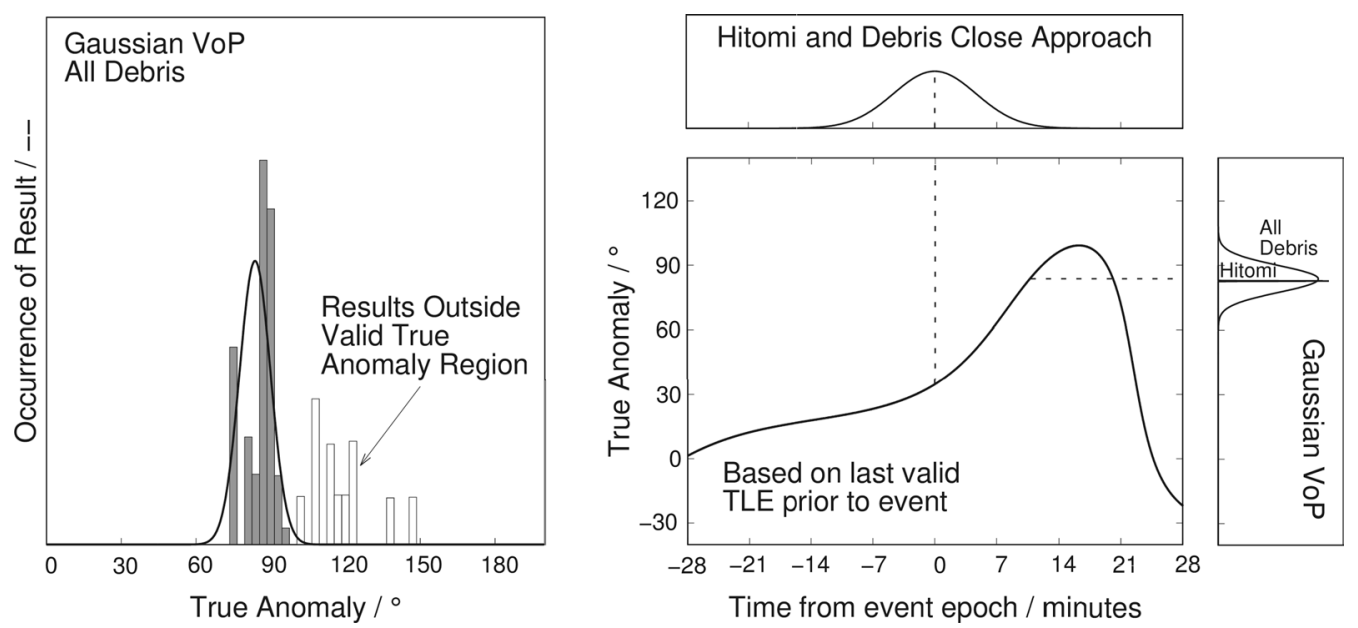

Fig. 3 Left Histogram of results from solving of Gaussian variation of parameters equations based on the last viable TLE before the event and five TLEs for each fragment (except for the two which decayed within a few days of the event). Right True anomaly of Hitomi predicted using SGP4 based on the last viable TLE before for the event. Normal probability density functions are given from close approach analysis for event time and from Gaussian VoP analysis for event true anomaly. The dotted lines indicate the mean values from the normal probability density functions

anomaly/epoch. From the collected results in Table 1, it can be seen that the mapping of the Gaussian VoP solutions (GD, GD + CA, GA and GA + CA) onto the time leads to asymmetric PDFs. Without knowledge of the accuracy of the true anomaly evolution, the results derived from the Gaussian VoP approach suggest an event epoch of March 26, 01:52 UTC with a worst case 99.7\% confidence time interval of less than 5 min which is roughly three times lower than that of the close approach methodology.

Several factors contribute to the credibility of the solution of the Gaussian VoP method: For one, the method has been shown to work for simulated cases without uncertainties. Furthermore, the mean solutions for the event true anomaly from Hitomi's main body and from the debris are very close together and finally they are both within the $+3 \sigma$ range of the estimated event epoch. As the uncertainties associated with Hitomi's true anomaly have not been assessed here, a definitive statement as to the absolute accuracy of the method would be premature.
In fact, such an analysis requires knowledge of the accuracy of given orbit data which is not available for TLEs.

Finally, the solutions for $\Delta v$ are below $10 \mathrm{~m} / \mathrm{s}$ in radial and in-track and below $33 \mathrm{~m} / \mathrm{s}$ in cross-track for all valid solutions. This again supports JAXA's assessment that this was not a high-energy fragmentation. For object 41337, the solving of the Gaussian VoP equations results in $\Delta v_{R} \approx+7.9 \mathrm{~m} / \mathrm{s}, \Delta v_{S} \approx+0.3 \mathrm{~m} / \mathrm{s}$ and $\Delta v_{W} \approx-1.4 \mathrm{~m} / \mathrm{s}$ which, applied at $v=82.72^{\circ}$, should lead to the observed change in osculating orbit elements.

\section{Event magnitude}

High-energy events commonly create substantial amounts of debris which are ejected into higher as well as lower orbits relative to the original one. Low-energy events typically only release a few objects and at low relative velocities. This behaviour can be observed by inspecting the debris clouds within the 'History of OnOrbit Satellite Fragmentations' (Johnson et al. 2008) shortly after the event epoch. When aerodynamic drag

Table 1 Mean event epoch $\left(t_{0}\right)$ and confidence interval limits from all methods

\begin{tabular}{|c|c|c|c|c|c|c|c|}
\hline Method & $-3 \sigma$ & $-2 \sigma$ & $-1 \sigma$ & $t_{0}$ & $+1 \sigma$ & $+2 \sigma$ & $+3 \sigma$ \\
\hline$C A$ & -14.00 & -9.33 & -4.67 & +00.00 & +4.67 & +9.33 & +14.00 \\
\hline GD & -3.58 & -2.45 & -1.28 & +10.95 & +1.55 & +3.72 & +4.97 \\
\hline $\mathrm{GD}+\mathrm{CA}$ & -3.32 & -2.20 & -1.12 & +10.13 & +1.23 & +2.68 & +4.62 \\
\hline GA & -0.05 & -0.03 & -0.02 & +10.51 & +0.02 & +0.03 & +0.05 \\
\hline $\mathrm{GA}+\mathrm{CA}$ & -0.05 & -0.03 & -0.02 & +10.51 & +0.02 & +0.03 & +0.05 \\
\hline
\end{tabular}

All values are given in minutes. The $t_{0}$ values relate to the mean estimated event time from the close approach (CA) method. All standard deviations relate to the $t_{0}$ value of the respective method. CA-close approach. GD-Gaussian VoP using all debris. GD + CA-combined result of close approach and Gaussian VoP using all debris. GA-Gaussian VoP using Hitomi main body only. GA + CA—combined result of close approach and Gaussian VoP using Hitomi main body only 
is a dominant force, the debris from a low-energy event quickly decay to altitudes below that of the original object. For high-energy events, this process takes significantly longer. Looking at the debris' orbit elements in the form of Gabbard diagrams [as in Johnson et al. (2008)] usually allows a quick assessment on whether a high- or a low-energy breakup occurred. Figure 4 shows the distribution of the fragments for April 1, just 6 days after the predicted event epoch, and for July 20 . Already on April 1, most of the fragments are at lower altitudes than the parent object (2016-012A). On July 20 , almost 4 months after the given initial TLEs, all of the fragments have lower orbital periods than the main object with two objects already having re-entered the Earth's atmosphere. These results support the previous assessment that this was likely not a high-energy event. It is therefore unlikely that many more large debris were created in the event than have already been identified.

\section{Conjunction analysis}

A conjunction analysis is performed using Electro-Optic Systems (EOS) Space Systems' conjunction analysis software and TLE data before and after the event to determine what catalogued objects were in the vicinity of Hitomi. This method is comparable to that of the Satellite Orbital Conjunction Reports Assessing Threatening Encounters in Space (SOCRATES) (Kelso and Alfano 2006).

Typically, the latest TLE is used in a routine all-on-all conjunction assessment. In this analysis, however, several all-on-all conjunction assessments are performed for epochs 23, 24 and 25 March 2016 and 1, 2 and 3 April 2016, i.e., excluding the erroneous TLEs. These results identify all objects with a close approach with Hitomi, and the whole catalogue of TLEs was downloaded each day between 20 March 2016 and 4 April 2016 to run the complete all-on-all conjunction assessment. The rationale behind this decision is to not miss a close approach by neglecting a valid TLE state.

An arbitrary error ellipsoid is assumed with dimensions [along track, cross track, radial] $=[2 \mathrm{~km}, 2 \mathrm{~km}, 1$ $\mathrm{km}]$ for all objects. These values are deemed descriptive of the error of a TLE state, and no considerations are made for nonlinear error state transition. For the calculation of the breakup time, only valid TLEs with epochs after the approximate breakup time are used to propagate backwards to estimate the time of the event.

\section{Close approaches}

Figure 5 shows the close approaches with Hitomi. The $y$ axis shows the calculated distance between the objects and Hitomi at the time of closest approach. The colour of the data points indicates the relative velocity of the approach. For each of the debris, multiple close approaches are displayed since multiple TLEs were used for each object in the analysis. This shows the variability that can occur in the conjunction assessments from different TLEs. An example of the issues that this variability causes is the missed Iridium-Cosmos collision [see Kelso (2009)].

In Fig. 5, the debris objects are identified with the remaining low-velocity close approaches (dark blue dots) corresponding to Hitomi debris that was catalogued at the time of the close approach analysis. This shows the problem faced by satellite operators by the debris environment. Five separate debris objects from the Fengyun $1 \mathrm{C}$ breakup appear in the analysis. Also present is debris from the Iridium-Cosmos collision. The estimated time of the breakup is indicated by the dashed vertical line.
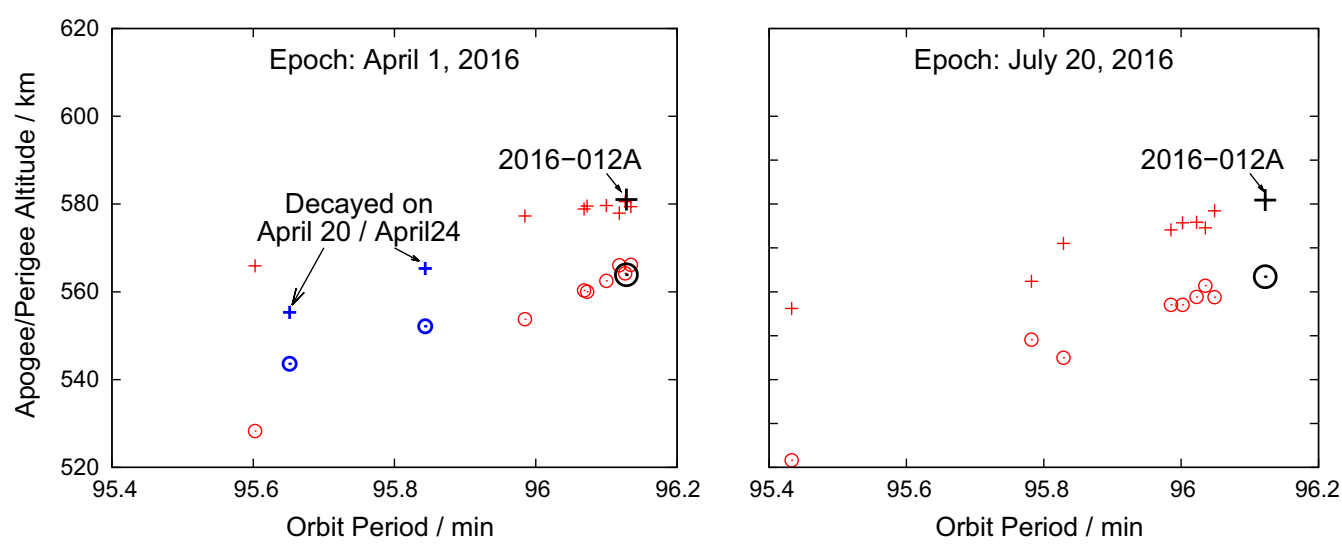

Fig. 4 Gabbard diagram of the Hitomi debris. Left first TLEs were published for April 1. Right TLEs for July 20. ' + ' indicate apogee altitude. ${ }^{\circ \prime}$ indicates perigee altitude. Black marks indicate Hitomi main body. Red marks indicate fragments still in orbit on July 20. Blue marks indicate fragments 41438 and 41443 which decayed end of April (see also Table 4) 


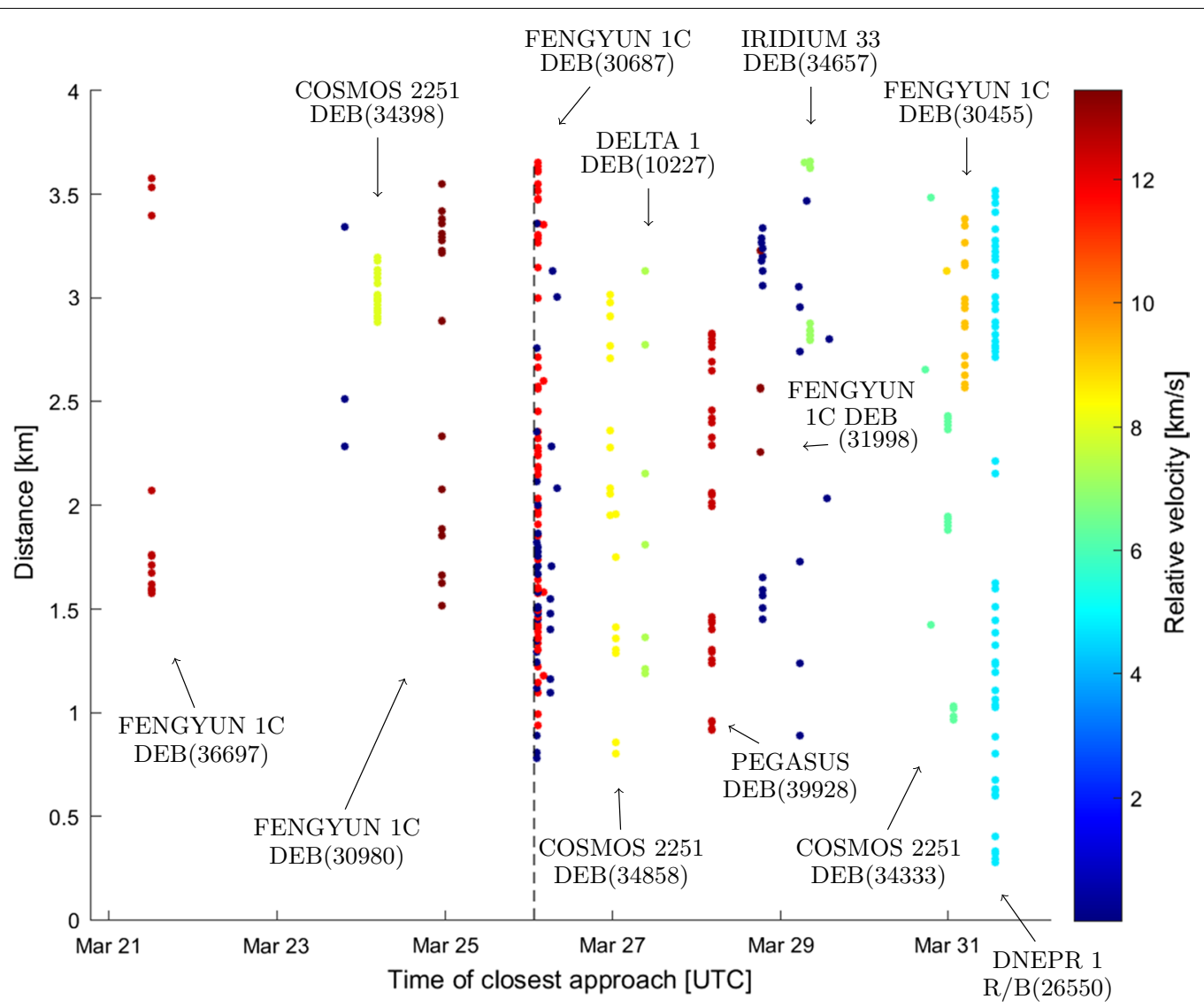

Fig. 5 Calculated close approach warnings for Hitomi. The close approaches resulting from debris objects are labelled with the NORAD ID in brackets. The dashed vertical line indicates the estimated breakup time

\section{Collision fragmentation simulation}

JAXA has surmised that the breakup of Hitomi was very likely caused by a combination of operational and design aspects (see 'Event description' section). In 'Event magnitude' section, a 'low-energy' cause for the breakup has been corroborated based on the number and orbit characteristics of the observed debris. Low-energy events can, however, also have other causes. In this section, the number of debris that would have been created if Hitomi had collided with one of the objects that had a close approach with Hitomi is estimated using the EVOLVE-4 breakup model (Johnson et al. 2001). Although the orbit evolution of these objects do not show any change that would suggest an actual collision with Hitomi, the analysis can be used to assess two things: (a) a collision with an object which is not included in the USSTRATCOM's public catalogue cannot be dismissed, if the number of fragments in any of the simulated conjunctions is similar to what has been observed for Hitomi; and (b) insight may be gained into what impact a catastrophic collision would have had on the environment.

Several assumptions are made to estimate the number of fragments that would be generated using a fragmentation model and compare them to the number that have been catalogued. The summary information for Hitomi is contained in Table 2.

The characteristics of the debris are estimated from a couple of sources. Firstly, the objects are assumed spherical. The ballistic coefficient, $B_{C}$, is defined as:

$$
B_{C}=\frac{C_{\mathrm{D}} A}{m} \quad\left(\mathrm{~m}^{2} / \mathrm{kg}\right)
$$

where $C_{\mathrm{D}}$ is the drag coefficient, $A$ is the constant cross-sectional area in the direction of motion and $m$ is the mass. The parameter $B_{C}$ is determined using EOS Space Systems' Ballistic Coefficient Estimation Method (BCEM) (Sang et al. 2013).

The cross-sectional area is chosen as the average value from historic radar cross-sectional (RCS) values. The RCS value is no longer published with the Satellite Situation Report, and a description of the new category system may be found here. ${ }^{1}$ Rearranging Eq. (1) and solving for $m$ gives:

\footnotetext{
${ }^{1}$ Space-Track.Org RCS Legend, https://www.space-track.org/documentation/loadLegendRCS, accessed 16-Apr-2016.
} 
Table 2 Hitomi satellite information from JAXA (2016) and orbit data from USSTRATCOM's Satellite Situation Report (SSR) retrieved on 6 May 2016

\begin{tabular}{ll}
\hline Parameter/unit & Value \\
\hline NORAD ID & 41337 \\
COSPAR ID & $2016-012 \mathrm{~A}$ \\
Name & Hitomi (ASTRO-H) \\
Mass $(\mathrm{kg})$ & 2700 \\
Period $(\mathrm{min})$ & 96.13 \\
Inclination $\left(^{\circ}\right)$ & 31.01 \\
Apogee altitude $(\mathrm{km})$ & 582 \\
Perigee altitude $(\mathrm{km})$ & 564 \\
RCS & 'LARGE' \\
Launch date & $2016-02-17$ \\
\hline
\end{tabular}

$$
m=\frac{C_{\mathrm{D}} A}{B_{C}} \quad(\mathrm{~kg}) .
$$

Assuming $C_{\mathrm{D}}=2.2, A=\mathrm{RCS}$ and $B_{C}$ is the value resulting from the BCEM method allows the mass to be estimated. The estimates for the debris objects are contained in Table 3.

The collisional energy $E$ may be calculated as:

$$
E=\frac{1}{2} \frac{m_{\mathrm{p}} m_{\mathrm{s}} v_{\mathrm{rel}}^{2}}{m_{\mathrm{p}}+m_{\mathrm{s}}} \quad(\mathrm{J})
$$

where $m_{\mathrm{p}}$ is the mass of the primary object $(\mathrm{kg}), m_{\mathrm{s}}$ is the mass of the secondary object $(\mathrm{kg})$ and $v_{\text {rel }}$ is the relative velocity $(\mathrm{m} / \mathrm{s})$. The energy-to-mass ratio EMR is:

$$
\mathrm{EMR}=\frac{E}{m_{\mathrm{S}}} \quad(\mathrm{J} / \mathrm{kg})
$$

The EMR threshold is set at 40,000 J/kg for a catastrophic collision. Assuming Hitomi as the primary object and the debris as the secondary object and using the information in Table 2, the mass calculated from (2) and the relative velocities found in the close approach analysis, we are able to calculate the EMR.

The number of fragments generated from this collision may be estimated from (Johnson et al. 2001), using the correction from (Krisko 2011):

$N\left(L_{c}\right)=\left\{\begin{array}{llll}0.1\left(\frac{m_{\mathrm{s}} \mathrm{v}_{\mathrm{rel}}^{2}}{100^{2}}\right)^{0.75} L_{c}^{-1.71}, & \text { if } & \mathrm{EMR}<40,000 & (\mathrm{~J} / \mathrm{kg}) \\ 0.1\left(m_{\mathrm{p}}+m_{\mathrm{s}}\right)^{0.75} L_{c}^{-1.71}, & \text { if } & \mathrm{EMR} \geq 40,000 & (\mathrm{~J} / \mathrm{kg})\end{array}\right.$

where $N\left(L_{c}\right)$ is the number of fragments larger than characteristic length $L_{c}$.

Substituting $L_{c}=0.1 \mathrm{~m}$ into Eq. (5), i.e., the approximate threshold size of the TLE catalogue, the number of objects larger than $10 \mathrm{~cm}$ generated from the collision can be estimated. The values are contained in Table 3.

The EVOLVE-4 model predicts that a conjunction with the lightest of the catalogued objects (34657 and 34858) would have produced a similar number of trackable debris as has been observed for the event. As previously stated, neither object show a change in orbit evolution which would indicate an actual collision with Hitomi. At an average mass of the two objects of about $45 \mathrm{~g}$ ( $40 \mathrm{~g}$ for 34657 and $51 \mathrm{~g}$ for 34858 ), a solid sphere made of sodium-potassium for instance would only have a diameter of $4.6 \mathrm{~cm}$ and would likely not be contained in the USSTRATCOM's catalogue. Liquid sodium-potassium was released at altitudes mostly around 900-950 km from Russian Buk reactors in the 1980s upon conclusion of their mission. They are believed to have formed spherules with sizes between $0.5 \mathrm{~mm}$ and $5.67 \mathrm{~cm}$ and have a density of about $0.9 \mathrm{~g} /$ $\mathrm{cm}^{3}$ (Wiedemann et al. 2011). These large spheres are expected to remain in orbit for decades, slowly decaying

\begin{tabular}{|c|c|c|c|c|c|c|}
\hline NORAD ID & Name & $B_{C}\left(\mathrm{~m}^{2} / \mathrm{kg}\right)$ & $\operatorname{RCS}\left(\mathrm{m}^{2}\right)$ & $v_{\text {rel }}(\mathrm{km} / \mathrm{s})$ & $m_{s}(\mathrm{~kg})$ & $N_{C}(>0.1)$ \\
\hline 10227 & DELTA 1 DEB & 0.0638 & 0.2603 & 7.331 & 8.9759 & $1.926^{*}$ \\
\hline 26550 & DNEPR 1 R/B & 0.0129 & 9.2720 & 4.738 & 1.581 & $2.714^{*}$ \\
\hline 30455 & FENGYUN 1CDEB & 0.0841 & 0.0282 & 9.176 & 0.7377 & 113 \\
\hline 30687 & FENGYUN 1C DEB & 0.5798 & 0.0195 & 11.766 & 0.0740 & 29 \\
\hline 30980 & FENGYUN 1CDEB & 0.5744 & 0.0127 & 13.436 & 0.0486 & 26 \\
\hline 31998 & FENGYUN 1C DEB & 0.6068 & 0.0118 & 13.283 & 0.0428 & 23 \\
\hline 34333 & COSMOS 2251 DEB & 0.3502 & 0.0354 & 6.211 & 0.2224 & 25 \\
\hline 34398 & COSMOS 2251 DEB & 0.2723 & 0.0317 & 7.976 & 0.2561 & 41 \\
\hline 34657 & IRIDIUM 33 DEB & 0.7428 & 0.0136 & 7.047 & 0.0403 & 8 \\
\hline 34858 & COSMOS 2251 DEB & 0.5923 & 0.0137 & 8.449 & 0.0509 & 13 \\
\hline 36697 & FENGYUN 1C DEB & 0.0734 & 0.0093 & 12.621 & 0.2787 & 88 \\
\hline 39928 & PEGASUS DEB & 0.1693 & 0.0164 & 12.496 & 0.2131 & 71 \\
\hline
\end{tabular}

Table 3 Results of simulated fragmentation with Hitomi and debris. $N_{C}(>0.1)$ number of fragments larger than $10 \mathrm{~cm}$ generated from the conjunction

$\mathrm{A}^{\prime * \prime}$ indicates that the conjunction was catastrophic (EMR $\geq 40,000 \mathrm{~J} / \mathrm{kg}$ ) 
in altitude over time. Steel or aluminium debris of similar mass may potentially have similar or smaller sizes, making them even harder to track. In conclusion, a collision with such a hard-to-track object could potentially produce a similar number of trackable debris objects. Two of the 12 modelled collisions are catastrophic with predicted fragments larger than $10 \mathrm{~cm}$ on the order of 2000-3000. This is similar to the number of fragments which have been catalogued from the breakup of the Fengyun 1C satellite (Wiedemann et al. 2014). As the relative velocities at these low altitudes are typically on the order of $10 \mathrm{~km} / \mathrm{s}$, a $1-\mathrm{cm}$ object is enough to end a satellite's mission. The Fengyun $1 \mathrm{C}$ debris cloud increased the spatial object density at the event altitude by roughly a factor of two for debris larger than $1 \mathrm{~cm}$. The relative increase in spatial object density and the resulting relative increase in close conjunctions at Hitomi's altitude would be much more pronounced due to the lower absolute spatial object density at those altitudes (compare Fig. 7). The increased aerodynamic drag in this region would, however, lead to a much faster decay of the debris, thereby alleviating the situation to a degree.

\section{Impact of Hitomi breakup on the environment}

There are different aspects to the impact of a fragmentation event on the debris environment. On the one hand, there is a very immediate effect as the new debris may collide with active spacecraft in the orbital vicinity, thereby endangering the success of their mission. On the other hand, as long as the debris is in Earth orbit, it can cause a catastrophic breakup of other large objects such as spacecraft or rocket bodies, which is the basis of the so-called Kessler syndrome.

Firstly, the remaining orbital lifetime of the Hitomi debris is estimated followed by an analysis of the potential contribution to the Kessler syndrome. Finally, the immediate impact of the event on active spacecraft and other intact objects (all satellites and rocket bodies) is assessed.

\section{Fragment orbit lifetime estimate}

The time until demise in Earth's atmosphere of the remaining fragments is estimated using the Orbital Spacecraft Active Removal (OSCAR) tool which is part of the European Space Agency's (ESA) Debris Risk Assessment and Mitigation Analysis Tool Suite (DRAMA). The orbit lifetime prediction utilises the FOCUS1 ('Fast Orbit Computation Utility Software 1') semi-analytic propagator which is also used in the creation of the debris population behind ESA's Meteoroid and Space Debris Terrestrial Environment Reference (MASTER). OSCAR supplies various means of solar and geomagnetic activity prediction. For the current analysis, OSCAR's implementation of the solar and geomagnetic prediction for the mean and $68.3 \%$ confidence interval $( \pm 1 \sigma)$ are used. The initial states for 22 August 2016 are supplied in the form of TLEs. The ballistic coefficients are derived from TLE's using EOS Space Systems' BCEM (see also 'Collision fragmentation simulation' section). It should be noted that the method's accuracy increases with the number of available TLE and that the results may change once more TLE become available. More importantly, however, Sang et al. (2013) found that based on the given data, the BC value using this method was consistently about an order of magnitude higher than the one obtained through the simple conversion $\mathrm{BC}=\left(12.741621 \cdot B^{*}\right)^{-1} \mathrm{~kg} / \mathrm{m}^{2}$ from Hoots and Roehrich (1980). The respective values are given in Table 4 . The size estimate supplied in the USSTRATCOM's Satellite Situation Report are also given in that table. All but two of the fragments are labelled as 'SMALL'. This indication means that their average radar cross-sectional (RCS) values are below $0.1 \mathrm{~m}^{2}$ which roughly relates to a size of $36 \mathrm{~cm}$. 'LARGE' objects have an RCS above $1 \mathrm{~m}^{2}$.

The two debris objects with the NORAD IDs 41438 and 41443 decayed within a month of the event. Figure 6 shows the results of the orbital lifetime estimate (left) alongside the predicted mean and $\pm 1 \sigma$ F10.7 radiation. The F10.7 radiation levels (10.7 cm wavelength) are a proxy for the Sun's extreme ultraviolet radiation which is the main driver for the atmospheric density variations at high altitudes and is the basis of orbital decay due to atmospheric drag. As can be seen, most of the debris is expected to have decayed by the next solar maximum around 2025. The main object, 41337, should remain on orbit for some decades.

Table 4 Ballistic coefficient of catalogued Hitomi fragments estimated using EOS Space Systems' BCEM (see also 'Collision fragmentation simulation' section)

\begin{tabular}{llll}
\hline Type & Object no. (NORAD ID) & Size (S/M/L) & $\boldsymbol{B}_{\mathbf{C}}\left(\mathbf{m}^{\mathbf{2}} \mathbf{k g}\right)$ \\
\hline HITOMI & 41337 & LARGE & 0.0007 \\
DEB & 41438 & SMALL & $>1$ \\
DEB & 41439 & SMALL & 0.079 \\
DEB & 41440 & SMALL & 0.136 \\
DEB & 41441 & SMALL & 0.113 \\
DEB & 41442 & LARGE & 0.025 \\
DEB & 41443 & SMALL & $>1$ \\
DEB & 41444 & SMALL & 0.124 \\
DEB & 41445 & SMALL & 0.128 \\
DEB & 41446 & SMALL & 0.366 \\
DEB & 41447 & SMALL & 0.117 \\
\hline
\end{tabular}

Size estimates are taken from USSTRATCOM's Satellite Situation Report 


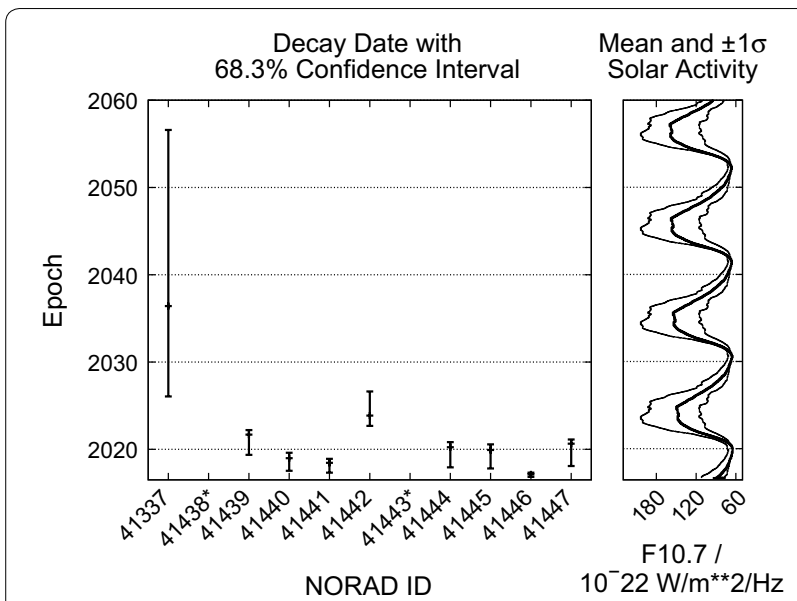

Fig. 6 Predicted orbital lifetime for all debris originating from Hitomi (left). The ${ }^{\prime * \prime}$ indicates objects which have already decayed. Error bars are given for the span of remaining orbital lifetime corresponding to the $68.3 \%$ confidence interval for the solar activity magnitude (right). Solar activity and remaining orbital lifetime predictions were performed using ESA's DRAMA software v2.0.1

\section{Contribution to Kessler syndrome}

With respect to the Kessler syndrome, the orbit inclination and altitude at which the fragments orbit Earth plays an important role. The overall collision risk is tied to the spatial object density, to the relative velocities and the remaining on-orbit time. Today, the regions in which the risk for a catastrophic collision is highest are the polar regions around $800 \mathrm{~km}$ altitude. In contrast to the GEO region where the general orbiting direction of all objects is the same (in the direction of Earth's rotation), the planes of the low Earth orbit (LEO) environment polar orbits are evenly spread out around the Earth. This causes the most likely impact direction on these orbits to be head-on with a relative velocity of $14-15 \mathrm{~km} / \mathrm{s}$. Large, massive spacecraft and rocket bodies which can no longer be controlled within this region are deemed as the most critical contributors: the probability for a collision is high and a conjunction will most likely lead to the creation of hundreds or even thousands of new debris which in turn may collide with other objects. Simulations such as those presented by Kebschull and Radtke (2014) have revealed that a number of Zenit-2 upper stages, ESA's Envisat, METOP-A and -B and also some Iridium spacecraft are among the objects with the highest 'environmental criticality' rating.

The distribution in spatial object density for the orbit altitude of interest is shown in Fig. 7. The orbit altitude of significant other spacecraft is indicated.

Hitomi has an orbit altitude of roughly $570 \mathrm{~km}$. It is therefore not located in the critical region. Atmospheric

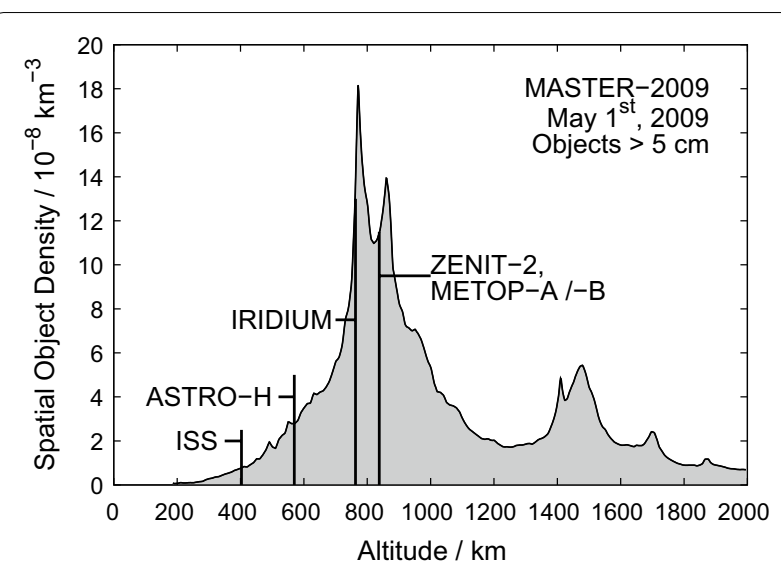

Fig. 7 Spatial object density for low Earth orbits for objects $5 \mathrm{~cm}$ and larger. The plot was created using ESA's MASTER-2009 software (v7.2). The spatial object density peak just below $800 \mathrm{~km}$ is created by the fragments from the two debris clouds of the Iridium 33 and Cosmos 2251 spacecraft which collided in early 2009. The peak just above that altitude is the debris from the Chinese anti-satellite test in which the meteorology satellite Fengyun 1C was deliberately destroyed by a ground-to-space missile (Liou and Johnson 2009; Johnson et al. 2007)

drag will cause the fragments to decay through other regions which are less densely populated than at $800 \mathrm{~km}$. This also means that the debris will never travel through the critical altitude band, and their contribution to the Kessler syndrome is low.

\section{Intact objects with potential close conjunction with Hitomi debris}

As can be seen in the Gabbard diagram (Fig. 4), the highest altitude of any fragment is about $580 \mathrm{~km}$ and atmospheric drag will continue to reduce their altitude over time. Only spacecraft which are orbiting at this altitude or lower could potentially collide with one of these fragments.

Transient objects A scan of the USSTRATCOM's SSR for 20 July 2016 reveals that about 600 intact objects are in Earth orbit with apogee above and perigee below $580 \mathrm{~km}$ altitude. For 10 May 2016, the Satellite Database of the Union of Concerned Scientists (UCS) (2016) contained 73 active spacecraft with these orbit characteristics. As long as objects spend most of their time above $580 \mathrm{~km}$, the statistical risk of colliding with one of the Hitomi fragments will be very low. They are therefore, at least statistically speaking, the ones which have the lowest nonzero probability for colliding with one of the Hitomi debris.

Objects below $520 \mathrm{~km}$ The SSR contains a total of about 340 intact objects with orbits entirely below $520 \mathrm{~km}$. The UCS list about 120 active spacecraft for this region. As 
these orbits are below the current altitude of the Hitomi fragments, a close conjunction may only occur in the future when the drag-induced decay causes the debris to pass through the orbital altitude regime of the listed intact objects. The most important spacecraft in this category is the International Space Station which currently is on a circular orbit just above $400 \mathrm{~km}$.

Objects in 'close orbital proximity' Confining the orbits to altitudes between 520 and $580 \mathrm{~km}$ results in a list of objects which are at the same altitude as the Hitomi debris is now. Their risk for a collision is immediately affected by the new debris. In this region, the SSR contains 59 intact objects with orbit information; 12 of these being upper stages and 47 being payloads. Of the rocket bodies, five (all are SL-3 R/B) were launched before 1990. For the same altitude band, UCS published 31 active spacecraft. A subset of the data for these is given in Table 5. In all, seven spacecraft are listed with launch masses in excess of one tonne. From the standpoint of the Kessler syndrome, these are the most critical objects among the active spacecraft, as a catastrophic collision involving one of these would create a large number of fragments. The most notable entry in this list, however, is the Hubble Space Telescope (HST) which has an orbit altitude of $560 \mathrm{~km}$. The collision velocity in the event of a conjunction would be lower than the $14-15 \mathrm{~km} / \mathrm{s}$ observed in polar orbits as the HST and Hitomi have very similar orbit inclinations $\left(28^{\circ}\right.$ and $31^{\circ}$, respectively). The exact value depends largely on the relative separation of the right ascension of ascending node. For similar values, the orbital planes will be roughly aligned and relative velocities will be very low. If they have a relative separation close to $180^{\circ}$, a conjunction near the line of nodes could occur in which one object is travelling on a shallow south-north trajectory while the other is moving in the opposite direction. This configuration would result in a collision velocity similar to the orbital velocity $(\cong 7 \mathrm{~km} / \mathrm{s})$.

\section{Conclusion}

The event time was estimated to March 26, 01:42 \pm 00:14 by calculating the minimum distance between the propagated states of the debris and of Hitomi. The location of the object at the estimated time of the event was compared to solutions for event locations obtained from solving of the Gaussian variation of parameters equations. Based on these results, the argument of latitude at the time of the event is estimated to have been $u \approx 83^{\circ}$. Solving of the equations revealed that radial, along-track and cross-track velocity changes in the order of 7.9, 0.3 and $-1.4 \mathrm{~m} / \mathrm{s}$ were imparted on the main body due to the fragmentation. By combining the normal probability density functions from the minimum distance approach and from the event location solutions, an event epoch which takes into account solutions in different parameter domains was obtained which places the event 10-11 min later which is well within the original estimate. This solution's accuracy in the time domain depends on the realism of the true anomaly evolution in the vicinity of the event epoch. The accuracy of this solution can therefore only be obtained if the accuracy of the orbit data upon which it relies is available. As this is unfortunately not the case, the absolute accuracy of this solution cannot be stated.

Due to the orbit altitude of the event, the debris from the Hitomi anomaly does not fuel the critical polar orbit region at altitudes around $800 \mathrm{~km}$. Of the eight fragments which remain in orbit, it was estimated that all but Hitomi's main body should deorbit before or during the upcoming solar maximum around 2025. The main body will likely remain in orbit for a few decades. Statistically, the probability that this object may be involved in a collision is much higher than for the fast decaying fragments.

The conjunction analysis revealed that a number of debris from the breakups of the Iridium 33, the Cosmos 2251 and the Fengyun 1C spacecraft were in Hitomi's orbital vicinity with potential for close conjunction. This observation highlights the impact of high-energy fragmentations on the operational safety of spacecraft in Earth orbit. Had one of these objects struck Hitomi's body, the expected number of debris would in most cases have exceeded the number of currently tracked objects for Hitomi. The catastrophic collisions between Hitomi and the Delta 1 debris or the Dnepr 1 rocket body especially would have been very damaging to the environment with thousands of debris larger than $10 \mathrm{~cm}$ being created. The simulations using the EVOLVE-4 model also showed that a non-catastrophic collision with a debris with a mass of about $45 \mathrm{~g}$ could potentially produce a similar number of large debris as have been catalogued. For spherical sodium-potassium debris released from a Russian Buk reactor, this would relate to a diameter of about $4.6 \mathrm{~cm}$. A steel or aluminium debris of similar mass would have similar or smaller sizes. As this size is below the USSTRATCOM's current catalogue threshold of about $10 \mathrm{~cm}$, the modelling results do not exclude a non-catastrophic collision with such an object. Just the same, JAXA's assessment that the breakup was a result of a combination of operational and design flaws is just as plausible. The current analysis does not allow any conclusion concerning the mechanism which caused the breakup.

A total of 59 intact objects are included in the USSTRATCOM's catalogue whose collision risk is immediately affected by the Hitomi debris. Of these 59 objects, 12 are rocket bodies, and 47 are active and inactive spacecraft. The most prominent active spacecraft which 
Table 5 List of active spacecraft within $520-580 \mathrm{~km}$ orbital altitude (UCS 2016)

\begin{tabular}{|c|c|c|c|c|c|c|}
\hline Int.-desig. & Name & Country & Perigee (km) & Apogee (km) & Incl. $\left(^{\circ}\right)$ & Launch mass $>1 \mathrm{t}$ \\
\hline $2015-049 \mathrm{~N}$ & XW-2B (CAS-3B) & China & 520 & 539 & 97.46 & \\
\hline 2015-049J & XW-2D (CAS-3D) & China & 520 & 539 & 97.46 & \\
\hline 2015-049R & KJSY-1 (Kongjian Shiyan-1) & China & 520 & 540 & 97.46 & \\
\hline 2015-049V & XC-1 (Xingchen 1) & China & 520 & 540 & 97.46 & \\
\hline 2015-049S & XC-2 (Xingchen 2) & China & 520 & 540 & 97.46 & \\
\hline 2015-049T & XC-3 (Xingchen 3) & China & 520 & 540 & 97.46 & \\
\hline 2015-049U & XC-4 (Xingchen 4) & China & 520 & 540 & 97.46 & \\
\hline 2015-049M & $X W-2 F(C A S-3 F)$ & China & 520 & 540 & 97.46 & \\
\hline 2015-049W & Zijing 1 & China & 520 & 540 & 97.46 & \\
\hline 2015-049K & LilacSat-2 & China & 520 & 541 & 97.47 & \\
\hline 2015-014A & Kompsat-3A & Sth Korea & 522 & 540 & 97.5 & \\
\hline 2006-029A & Genesis-1 & USA & 522 & 569 & 64.5 & * \\
\hline 2015-077E & Galassia & Singapore & 529 & 549 & 14.98 & \\
\hline 2015-077C & Athenoxat-1 & Singapore & 532 & 550 & 14.98 & \\
\hline 2015-077A & Velox C1 & Singapore & 533 & 550 & 14.98 & \\
\hline 2015-077B & Kent Ridge 1 & Singapore & 534 & 551 & 14.98 & \\
\hline 2015-077D & TeLEOS 1 & Singapore & 535 & 550 & 15 & \\
\hline $2002-004 A$ & HESSI (RHESSI) & USA & 535 & 551 & 38 & \\
\hline 2013-042A & Kompsat-5 & Sth Korea & 535 & 552 & 97.6 & * \\
\hline 2015-077F & Velox 2 & Singapore & 537 & 550 & 14.98 & \\
\hline 2008-029A & GLAST & USA & 537 & 556 & 25.6 & * \\
\hline 2007-006E & Falconsat-3 & USA & 538 & 540 & 35.4 & \\
\hline 2012-017A & RISat-1 & India & 538 & 541 & 97.6 & * \\
\hline 2007-006F & CFESat & USA & 538 & 544 & 35.4 & \\
\hline 2007-015A & AIM & USA & 544 & 552 & 97.9 & \\
\hline $2005-025 A$ & Suzaku (Astro E2) & Japan/USA & 548 & 558 & 31.4 & * \\
\hline 2013-015E & BeeSat-3 & Germany & 554 & 581 & 64.8 & \\
\hline 1990-037B & Hubble Space Telescope & ESA/USA & 555 & 559 & 28.5 & * \\
\hline 2013-015G & BeeSat-2 & Germany & 555 & 579 & 64.8 & \\
\hline $2006-021 \mathrm{~A}$ & Resurs-DK1 & Russia & 564 & 571 & 69.9 & * \\
\hline 2001-007A & Odin & Sweden & 569 & 573 & 97.6 & \\
\hline
\end{tabular}

is affected by the debris is the Hubble Space Telescope whose orbit is just $10 \mathrm{~km}$ below that of Hitomi. Other active spacecraft with significant mass which are affected include the inflatable Genesis-1 habitat, Kompsat-5, GLAST, RISat-1, Suzaku and Resurs-DK1. The draginduced gradual decrease in orbit altitude of the Hitomi fragments will cause these objects to pass through the orbit regime of other, low-orbiting spacecraft, whereby new close conjunctions may occur. One of these objects is the International Space Station.

\section{Abbreviations}

ACS: attitude control system; BCEM: ballistic coefficient estimation method; DRAMA: Debris Risk Assessment and Mitigation Analysis; EOB: extensible optical bench; EOS: Electro-Optic Systems; ESA: European Space Agency; HST: Hubble Space Telescope; intact: active and passive spacecraft and rocket bodies; JSPOC: Joint Space Operations Center; JST: Japan Standard Time; LEO: Iow Earth orbit; OSCAR: Orbital Spacecraft Active Removal; PDF: probability density function; RCS: radar cross section; RW: reaction wheel; SERC:
Space Environment Research Centre; SGP4: Simplified General Perturbations 4; SOCRATES: Satellite Orbital Conjunction Reports Assessing Threatening Encounters in Space; SSR: Satellite Situation Report; TLE: two-line element; USSTRATCOM: United States Strategic Command; UTC: Universal Time Coordinated; VoP: variation of parameters.

Parameters

a: semi-major axis; e: eccentricity; i: inclination; $\Omega$ : right ascension of ascending node; $\omega$ : argument of perigee; $v$ : true anomaly; $u$ : orbit angle; $v$ : velocity; $\Delta v$ : velocity change.

\section{Authors' contributions}

SF performed the event location analysis and the impact on the environment analysis. He aided in the design of the study and performed final editing and submission of the manuscript. JB carried out the event time estimated and conjunction analysis and drafted the first version of the manuscript. ML analysed the radar cross section of the debris, inferred object sizes used in the conjunction analysis and was instrumental in the conjunction analysis results evaluation. MM is in charge of developing the GPU-based conjunction assessment software and developed software which was key for the event location analysis. CS initiated the study, participated in the design of the study and helped to draft the manuscript. All authors read and approved the final manuscript. 


\begin{abstract}
Author details
1 Space Environment Research Centre (SERC) Limited, Weston Creek, ACT 2611, Australia. ${ }^{2}$ Electro Optical Systems (EOS), Weston Creek, ACT 2611, Australia.
\end{abstract}

\section{Acknowledgements}

The authors would like to acknowledge the support of the Cooperative Research Centre for Space Environment Management (SERC Limited) through the Australian Government's Cooperative Research Centre Programme. Furthermore, the authors wish to thank the reviewers of the manuscript for their insightful and helpful comments.

\section{Competing interests}

The authors declare that they have no competing interests.

\section{About SERC}

To help address the issues involved with safe spacecraft operations in the presence of space debris, the CRC for Space Environment Management, managed by the Space Environment Research Centre (SERC), has been established (www.serc.org.au). Key developments are being made in the positioning accuracy especially for GEO and HEO objects through the application of adaptive optics astrometry wherein atmospheric distortions are removed through adaptive optics. Attitude estimation from light curve analyses will allow incorporating non-spherical object geometries into orbit determination and prediction which will also aid in decreasing the uncertainty in conjunction assessments. By developing operational software which is designed to run on a single CPU, parallelised on multi-core CPUs or massively parallelised on GPUs while retaining double precision accuracy, high-accuracy approaches which would ordinarily not be feasible due to long processing times can be employed routinely. It was shown in previous research that orbital propagation can benefit greatly from massively parallel hardware architectures such as graphics processors (Möckel 2015). A core effort within SERC is to implement a high-accuracy all-on-all conjunction assessment for large object catalogues in this framework which is expected to reduce the required processing time considerably. SERC is presently in the process of setting up its first self-sustained catalogue of LEO to GEO objects which will be maintained using multiple passive and active optical instruments and in-house developed scheduling software for optimal sensor tasking. One of the outputs SERC is working towards is the ability to provide high accuracy conjunction assessments so as to help reduce the number of collision avoidance manoeuvres. Furthermore, an active ground-based laser collision avoidance system using photon pressure is being developed which will support debris mitigation measures.

\section{Publisher's Note}

Springer Nature remains neutral with regard to jurisdictional claims in published maps and institutional affiliations.

Received: 7 September 2016 Accepted: 4 April 2017

Published online: 18 April 2017

\section{References}

Flegel S, Gelhaus J, Möckel M, Wiedemann C, Kempf D, Krag H, Vörsmann P (2011) Maintenance of the ESA MASTER model-final report. Technical Report ESA Contract Number: 21705/08/D/HK, European Space Agency Hoots FR, Roehrich RL (1980) Spacetrack report no. 3-models for propagation of NORAD elements sets. Spacetrack Report, vol 3(3)

Inter-Agency Space Debris Coordination Committee-Working Group 2 (2013) Stability of the future LEO environment. Technical Report Action Item 27.1, Inter-Agency Space Debris Coordination Committee

JAXA (2016) Hitomi experience report: investigation of anomalies affecting the x-ray astronomy satellite "Hitomi" (ASTRO-H). Press release, JAXA, 31 May 2016. http://global.jaxa.jp/projects/sat/astro_h/files/topics_20160531.pdf
Johnson NL, Krisko PH, Liou JC, Anz-Meador PD (2001) NASA's new breakup model of EVOLVE 4.0. Adv Space Res 28(9):1377-1384. doi:10.1016/ s0273-1177(01)00423-9

Johnson NL, Stansbery E, Liou J-C, Horstman M, Stokely C, Whitlock D (2007) The characteristics and consequences of the break-up of the Fengyun-1C spacecraft. In: Proceedings of the 58th international astronautical congress. International Astronautical Federation, Sept 2007. Paper Number IAC-07-A6.3.01

Johnson NL, Stansbery E, Whitlock DO, Abercromby KJ, Debra S (2008) History of on-orbit satellite fragmentations, 14th edn. Technical Report NASA/ TM-2008-214779, Orbital Debris Program Office, National Aeronautics and Space Administration

Kebschull C, Radtke J (2014) Deriving a priority list based on the environmental criticality. In: International astronautical congress, Sept 2014 IAC-14,A6,P,48x26173

Kelso TS, Alfano S (2006) Satellite orbital conjunction reports assessing threatening encounters in space (SOCRATES). In: Proceedings of SPIE, 6221, modeling, simulation, and verification of space-based systems, vol III, $p$ 622101. doi: $10.1117 / 12.665612$

Kelso TS (2009) Analysis of the Iridium 33-Cosmos 2251 collision. Adv Astron Sci 135(suppl 2):1099-1112

Kessler DJ (1991) The limits of population growth in low Earth orbit. Adv Space Res 11(12):63-66

Krisko P (2011) Proper implementation of the 1998 NASA breakup model. Orbital Debris Q News 15(4):4-5

Liou J-C, Johnson NL (2009) Characterization of the cataloged Fengyun-1C fragments and their long-term effect on the LEO environment. Adv Space Res 43:1407-1415. doi:10.1016/j.asr.2009.01.011

Möckel M (2015) High performance propagation of large object populations in earth orbits. Ph.D. thesis, Technische Universität Carolo-Wilhelmina zu Braunschweig. doi:10.5281/zenodo.48180

Sang J, Bennett JC, Smith CH (2013) Estimation of ballistic coefficients of low altitude debris objects from historical two line elements. Adv Space Res 52(1):117-124. doi:10.1016/j.asr.2013.03.010

UCS (2016) Union of Concerned Scientists Satellite Database. Retrieved May 2016. https://s3.amazonaws.com/ucs-documents/nuclear-weapons/satdatabase/2-25-16+update/UCS_Satellite_Database_1-1-16.xls

Vallado DA, McClain WD (2013) Fundamentals of astrodynamics and applications. Space Technology Library, 4 edn. ISSN:9781881883203

Wiedemann C, Flegel S, Gelhaus J, Krag H, Klinkrad H, Vörsmann P (2011) NaK release model for MASTER-2009. Acta Astron 68:1325-1333

Wiedemann C, Flegel S, Kebschull C (2014) Additional orbital fragmentation events. In: Proceedings of 65th international astronautical congress, 2014. IAC-14.A6.P.57

\section{Submit your manuscript to a SpringerOpen ${ }^{\circ}$ journal and benefit from:}

- Convenient online submission

- Rigorous peer review

- Immediate publication on acceptance

- Open access: articles freely available online

- High visibility within the field

Retaining the copyright to your article

Submit your next manuscript at springeropen.com 